\title{
Radiation Protection in PET Room
}

\author{
Ü. KARA ${ }^{a, *}$, H.O. TEKIN ${ }^{b}$ AND İ. AKKURT ${ }^{c}$ \\ ${ }^{a}$ Suleyman Demirel University, Vocational School of Healt Services, Isparta, Turkey \\ ${ }^{b}$ Usküdar University, Vocational School of Healt Services, Istanbul, Turkey \\ ${ }^{c}$ Suleyman Demirel University, Physics Department, Isparta, Turkey
}

\begin{abstract}
Applications of radiation in medicine is based on the radiation ability of destroing the tumor cells and creating the images of internal organs. This two features play an important role in the diagnosis and treatment of diseases by radiation. In medicine X-ray and radionuclides being sources of gamma rays as $\mathrm{Tc}^{99 m}, \mathrm{I}^{137}$, or $\mathrm{Tl}^{201}$ are used for the diagnosis of diseases, as beta particles uses for the treatment of diseases. In nuclear medicine radioactive isotopes are built into pharmaceuticals and create radiopharmaceuticals. Radiopharmaceutical administered to patient gathers in examined organ and gives information on functional and anatomical status of the organ. Radiation workers prepare radiopharmaceuticals, transport it, implement, take diagnostic images and survey the therapy. Their radiological protection is very important. Efficient radiation protection can be achieved by factors of time (reduce the time spent close to radioactive source to minimum), distance (keep maximal distance to radioactive source), and shielding (sufficient amount of protective material placed between the source and body) concepts are the basis.
\end{abstract}

DOI: $10.12693 /$ APhysPolA.128.B-375

PACS: 87.50.yk, 87.57.uk

\section{Introduction}

$\mathrm{PET} / \mathrm{CT}$ medical imaging is relatively new imagin technique. Positron emission tomography is sort of nuclear medicine imaging. It covers all organs of the body, delivering morphological and functional informations in a comprehensive way. In nuclear medicine imaging department small amounts of radioactive materials serve in diagnosis. Radiotherapy treats a variety of diseases, most head and neck tumors as well as curing other sensitive areas: lungs, breast, colorectal, esophagus, stomach. Radiotracers are used to investigate the presence of cancer cells in the thyroid or pancreas and other abnormalities within the body. Oncologic PET/CT imaging is applicable in most common types of cancer: lung cancer, head and neck tumors, colon and rectal cancer, breast cancer, thyroid cancer etc. [1]. In Turkey the most common radioactive material used in $\mathrm{PET} / \mathrm{CT}$ is fluorine-18 deoxyglucose (FDG). Glucose as the important energy source is biodistributed to all tissues, allowing FDG to display all organs of the body. Tumor tissues have higher density and faster metabolism than normal tissues. FDG oncologic PET/CT activities for the diagnosis and treatment of cancer, that has become one of the most important therapeutical modalities, are based on the principle. Neurological PET/CT, such as the Alzheimer disease diagnostics significantly raisees the quality of life, due to diagnosis of early stages of dementia and other neurological ailments. PET/CT scan measures important body functions, such as blood flow, oxygen consumption, and sugar (glucose) metabolism. It allows to the evaluation

*corresponding author; e-mail: umitkara@sdu.edu.tr of condition of organs and tissues and its functioning. In contemporary cancer research, the most commonly used method PET/CT imaging technology is a technique being the merger of two techniques, intravenous administration of FDG for acumulation it in the tumor cells and using radiotracers to monitor the tumor cells. FDG concentraiting even in the smallest cancer lumps at an early stage enables fast diagnosis. For making PET/CT profitable the attentiveness for the side effects of radiation on health of patients is necessary. This paper presents a brief overlook of dosimetry methods related to PET/CT technology and to the methods of radiation protection $[2,3]$.

\section{Material and method}

Gathering data for this paper we worked at the Nuclear Medicine Department in Suleyman Demirel Research Hospital, where PET/CT apparatus Philips GEMINI is installed. We collect data on irradistion of nuclear medicine patients, staff and doctors involved in PET/CT therapy and diagnostics.

\section{Conclusion}

In this study the effectivness of radiation shielding has been tested. It is the most widely used mode of protection for activities in nuclear medicine, in contact with the radiopharmaceuticals and other radioactive material, including fluorine-18 deoxyglucose. We measured photon dose values in a $\mathrm{PET} / \mathrm{CT}$ room. We concluded that the measured photon dose depends significantly on radiation shielding. Irradiation dose and dose equivalent rate has been measured by means of Geiger-Müller counter and the Polimaster 1703MO-1b gamma spectroscopic dosimeter. Results were taken in different distance from the 

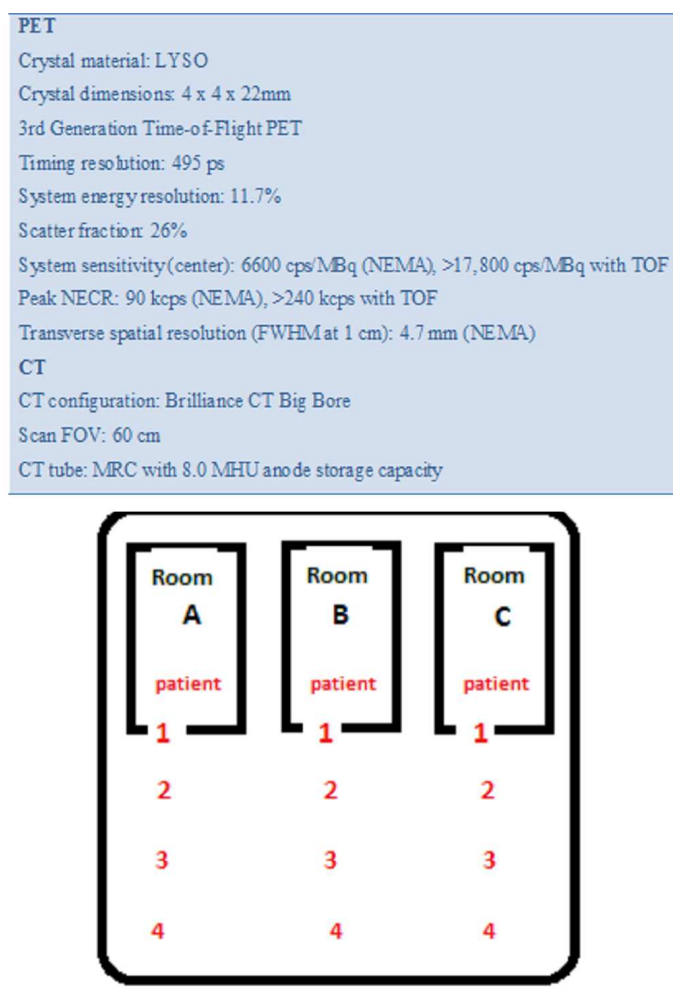

Fig. 1. Plan of PET/CT and localization of measuring points.

TABLE

The $\mathrm{PET} / \mathrm{CT}$ radiation measurement in consecutive points. "+" — shielding, "-" — without shielding.

\begin{tabular}{c|c|c|c|c|c|c|c|c|c|c}
\hline \hline \multirow{2}{*}{ PET/CT } & {$\left[\frac{\mathrm{mR}}{\mathrm{h}}\right]$} & {$\left[\frac{\mu \mathrm{Sv}}{\mathrm{h}}\right]$} & {$\left[\frac{\mathrm{mR}}{\mathrm{h}}\right]$} & {$\left[\frac{\mu \mathrm{Sv}}{\mathrm{h}}\right]$} & {$\left[\frac{\mathrm{mR}}{\mathrm{h}}\right]$} & {$\left[\frac{\mu \mathrm{Sv}}{\mathrm{h}}\right]$} & {$\left[\frac{\mathrm{mR}}{\mathrm{h}}\right]$} & {$\left[\frac{\mu \mathrm{Sv}}{\mathrm{h}}\right]$} \\
\cline { 2 - 9 } & $d=200 \mathrm{~cm}$ & \multicolumn{2}{|c|}{$d=300 \mathrm{~cm}$} & \multicolumn{2}{|c|}{$d=400 \mathrm{~cm}$} & \multicolumn{2}{l|}{$d=500 \mathrm{~cm}$} \\
\hline+ & 0.1 & 0.8 & 0.05 & 0.7 & 0.03 & 0.6 & 0.02 & 0.5 \\
- & 2 & 3.5 & 1.4 & 2.45 & 0.15 & 1.3 & 0.06 & 0.7 \\
+ & 0.06 & 0.7 & 0.03 & 0.6 & 0.01 & 0.55 & 0.02 & 0.5 \\
- & 0.6 & 4.15 & 0.3 & 3.7 & 0.15 & 1.8 & 0.05 & 0.6 \\
+ & 0.01 & 0.9 & 0.07 & 0.8 & 0.04 & 0.7 & 0.03 & 0.6 \\
- & 0.7 & 4 & 0.4 & 2.2 & 0.1 & 2 & 0.05 & 0.6
\end{tabular}

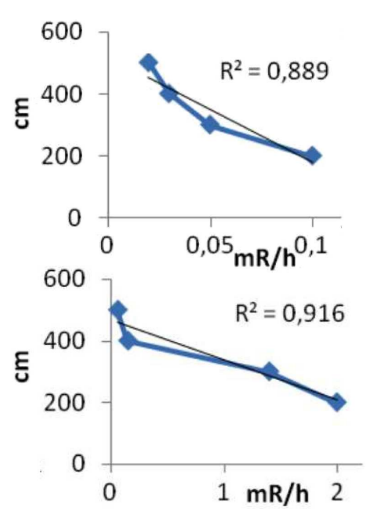

Fig. 2. Radiation dose in PET/CT room B with (left) and without (right) shielding.

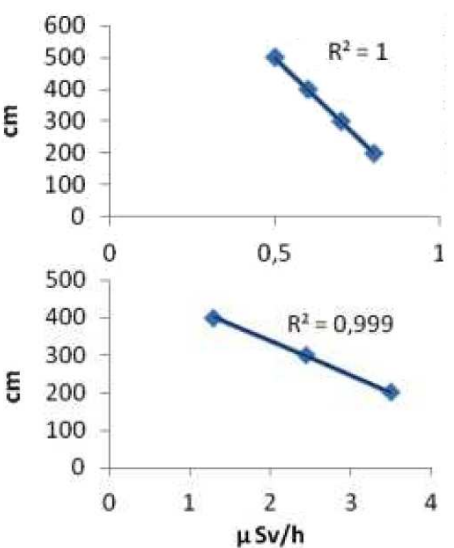

Fig. 3. Results for measuring points at PET/CT rooms A (left) and B (right).


Fig. 4. Change of dose with distance.
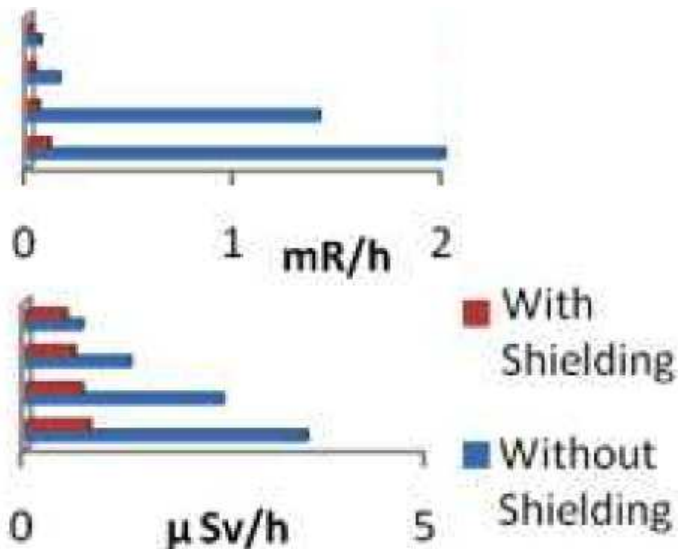

Fig. 5. The diagram of radiation changes in PET/CT room with distance. 
source, with and without shielding materials. Clinic in and out waiting rooms were tested also.

To protect yourself and the patient from unintended exposure to radiation following measures can be taken by employees $[4,5]$ :

- keep radiation sources within shielded enclosures whenever exposure is not necessary,

- maintain adequate distance from exposed radiation sources,

- reduce exposure time to a minimum,

- Wear protective clothing (lead apron, etc.).

Rules for patients care are:

- the physician is responsible for protecting the patient from unnecessary radiation,

- use the pediatric mode for children,

- observe the safety recommendations while performing a scanning procedure on a patient,

- never leave a patient unobserved before, during, or after a procedure.
Generally speaking the golden rule ALARA, keep the level "as low as reasonably achievable" should not be overlooked.

\section{References}

[1] T.M. Blodgett, C.C. Meltzer, D.W. Townsend, Radiology 242, 360 (2007).

[2] P.E. Kinahan, B.H. Hasegawa, T. Beyer, Semin. Nucl. Med. 33, 166 (2003).

[3] D.W. Townsend, J.P. Carney, J.T. Yap, N.C. Hall, JACR 3, 55 (2009); J. Nucl. Med. 45, 4S (2004).

[4] The 2007 Recommendations of the International Commission on Radiological Protection, Ann. ICRP 37, 1 (2007).

[5] European Guidance on Estimating Population Doses from Medical Ray Procedures, Radiation Protection 154, European Commission, Luxembourg 2008. 